\title{
A tissue atlas of ulcerative colitis to guide TNF inhibitor therapy
}

\section{Aaron Mayer}

Stanford University School of Medicine

\section{Derek Holman}

Stanford University

Utkarsh Tandon

Enable Medicine

\section{Salil Bhate}

Department of Microbiology \& Immunology, Stanford University School of Medicine

\section{Sunil Bodapati}

Enable Medicine

\section{Graham Barlow}

Stanford University

\section{Jeff Chang}

Enable Medicine

\section{Sarah Black}

Stanford University

\section{Erica Crenshaw}

Stanford University

\section{Brigid Boland}

University of California San Diego

\section{Sarah Streett}

Stanford University

\section{William Sandborn}

UCSD

\section{John Chang}

University of California San Diego

\section{Sanjiv Gambhir}

Stanford University https://orcid.org/0000-0002-2711-7554

\section{Garry Nolan}

Stanford School of Medicine https://orcid.org/0000-0002-8862-9043

\section{Christian Schürch}

Stanford University https://orcid.org/0000-0002-1792-1768

Stephan Rogalla ( $\sim$ srogalla@stanford.edu ) 


\section{Biological Sciences - Article}

Keywords: Ulcerative colitis, cellular neighborhoods, CODEX, multiplexed imaging, tissue architecture, TNF inhibitors, therapy prediction, autoimmune disease

Posted Date: February 4th, 2021

DOl: https://doi.org/10.21203/rs.3.rs-167888/v1

License: (c) (1) This work is licensed under a Creative Commons Attribution 4.0 International License. Read Full License 


\section{Abstract}

Ulcerative colitis is a chronic-relapsing inflammatory disease of the large intestine with a complex, multifactorial pathogenesis. TNF inhibitors are widely used to suppress immune-mediated tissue damage in ulcerative colitis patients; however, therapy failures are common. Predicting TNF inhibitor response requires an understanding of the architectural features that underlie mucosal inflammation and those responsible for resistance. Here, we used highly multiplexed immunofluorescence to uncover the spatially resolved tissue architectures underlying disease progression and treatment response in 42 tissue regions from 34 individuals. We created a tissue atlas and performed spatial analysis to identify cell-cell contacts and cellular neighborhoods. We observed that cellular functional states depend on cellular neighborhood and that a subset of inflammatory cell types and cellular neighborhoods in ulcerative colitis patients persisted even during treatment with TNF inhibitor, indicating resistant niches. A computer vision model, with no a priori assumptions regarding cellular architectural features, was able to predict TNF inhibitor resistance. This spatial model significantly outperformed classification models based on single-cell data alone. Our results demonstrate the value of a spatial tissue atlas as a precision medicine tool to guide treatment of patients suffering from autoimmune diseases.

\section{Introduction}

Ulcerative colitis (UC), a chronic-relapsing inflammatory bowel disease (IBD), is one of the most common autoimmune disorders ${ }^{1,2}$. UC results from a dysregulated interplay of genetic, immune, environmental, and microbiome factors ${ }^{1-3}$. UC typically persists for decades, resulting in high medical costs, diminished workplace productivity, and significantly impaired quality of life. Due to the chronic inflammation associated with UC, patients have a 1.5-times higher long-term risk for colorectal cancer compared to healthy individuals ${ }^{4,5}$. In recent years, novel treatment options for UC have been introduced, expanding the therapeutic standard of care from broadly immunosuppressive monotherapies, (i.e., corticosteroids, azathioprine, and methotrexate) to include more targeted immunomodulatory drugs.

Tumor necrosis factor inhibitors (TNFi) such as infliximab and adalimumab have become frontline targeted therapies for $\mathrm{UC}$ and have led to increased rates of sustained remission and decreased rates of surgery 6,7 . These inhibitors have fewer side effects than standard immunosuppressants and the incidence of chronic inflammation-induced colorectal cancer is reduced in TNFi-treated patients; however, TNFi-treated patients have an increased risk for opportunistic infections and for some malignancies including lymphomas, acute myeloid leukemias, myelodysplastic syndromes, and skin carcinomas ${ }^{8}$. Furthermore, primary non-response to TNFi therapy occurs in $13-40 \%$ of UC patients, and loss of response or adverse effects are reported in up to $46 \%$ of the remaining patients within 12 months of therapy initiation ${ }^{9-12}$. Moreover, the introduction of TNFi to standard UC treatment regimens results in significantly increased costs. Combined direct and indirect costs caused by the disease is estimated at $\$ 45$ billion per year in the United States and Europe ${ }^{1}$. Additional biologics, such as vedolizumab, an antibody that antagonizes a $4 \beta 7$ integrins, have been approved for treatment of patients with UC, but 
identification of patients who will respond to TNFi remains an unmet clinical challenge ${ }^{6,13}$. Predictive biomarkers to identify the best therapy for each individual patient at the time of diagnosis are lacking ${ }^{12-}$ 14 .

The success of clinical and biological phenotyping to identify novel biomarkers to guide UC therapy has been limited. The fecal proteins calprotectin and lactoferrin, which are released by activated neutrophils in the gut, are useful in monitoring disease activity but have no role in the decision making process with regards to a specific treatment ${ }^{7,15}$. Serological markers such as C-reactive protein and anti-neutrophil cytoplasmic antibodies were investigated as therapy predictors in Crohn's disease but data were inconclusive. Studies suggest that the only reliable clinical predictor of TNFi therapy failure in IBD was the presence of strictures in Crohn's disease as assessed endoscopically or radiologically; however, this may be an indicator of disease severity and complications rather than underlying disease biology ${ }^{16}$. Therefore, recent studies have aimed at enabling precision medicine in IBD through the use of new technologies like single-cell RNA sequencing (scRNA-seq) and mass cytometry to predict therapy response and disease activity ${ }^{17-19}$. scRNA-seq analysis in a cohort of Crohn's disease patients with ileal disease was able to identify pathogenic cellular modules that were weakly, although significantly, associated with resistance to TNFi therapy ${ }^{18}$. The presence of these immune cell modules suggests a spatial and functional relationship between the underlying cell types, but spatial information is lost during the preparation of samples for scRNA-seq so this has not been tested.

Imaging technologies capable of spatially mapping immune cell states within tissue at single-cell resolution should improve our understanding of how cellular interactions and gut remodeling contribute to UC pathogenesis and TNFi resistance. Multiplexed imaging tools are revolutionizing the development of biomarker-based diagnostics and have led to novel therapeutic insights in other disease settings ${ }^{20}$. Imaging mass cytometry has been used to better understand the high-dimensional tissue pathology in breast cancer patients and the changing cell populations associated with diabetes disease progression 21,22 . A multiplexed immunofluorescence-based imaging technology called co-detection by indexing (CODEX) has allowed simultaneous visualization of up to 60 biomarkers on intact tissue with single-cell resolution ${ }^{23,24}$. These biomarker panels can be custom designed and utilized to elucidate cell types and their functional states. The spatial interplay between these markers can be used to identify cellcell interactions and cellular neighborhoods (CNs). In colorectal cancer, these spatial features have revealed coordinated $\mathrm{CNs}$ underlying antitumoral immunity and disease progression. These changes in the tumor-immune microenvironment were correlated with patient outcomes but were not visible as changes at the single-cell level ${ }^{24}$.

Here, we used CODEX to create the first spatial atlas of the gut and its rewiring during UC pathogenesis. In 42 biopsy regions taken from a cohort of 34 individuals, we analyzed 52 biomarkers on 1,710,973 spatially resolved single cells to identify 13 conserved cell types. We mapped changes in frequency and functional states during inflammation and in response to TNFi treatment. We utilized mathematical frameworks to assess cellular contacts and neighborhoods, and we correlated these features with clinical 
phenotypes. This allowed us to identify cellular niches that were resistant to TNFi therapy. Furthermore, we developed a computer vision model, based on our spatial cell maps, that classified TNFi responders and non-responders with high accuracy $(A \cup C=0.947)$. To disseminate our results, we created a cloudbased platform called the Explorer that allows users to search our spatial maps for additional insights into UC. Our research suggests that examining cells within their spatial context is paramount to identifying new biomarkers of therapy response in $\mathrm{UC}$ and that this framework has the potential to improve our understanding of inflammatory immune diseases.

\section{Results}

\section{A spatial atlas of the UC inflammatory microenvironment}

We used CODEX to characterize the inflammatory microenvironment of the colon in 42 tissue biopsy regions from 29 UC patients and 5 healthy controls. Fifteen UC patients were being treated with TNFi at the time of biopsy (Figure S1a and Table S1). Long-term clinical follow-up was available for all patients, allowing categorization of the patients into TNFi responders vs. non-responders following the practice guidelines of the American Gastroenterological Association (AGA) for moderate to severe UC ${ }^{7}$. In line with previous reports ${ }^{9,25}$, in our cohort, $~ 50 \%$ of TNFi-treated patients did not subsequently respond to TNFi therapy (Table S1). We imaged multiple tissue regions per sample with a 52-plex CODEX antibody panel including markers for immune, epithelial, and stromal cell components of the intestine as well as functional markers such as 4-1BB (also known as TNFRSF9 or CD137), IL-6R (also known as CD126), and TNFR2 (also known as CD120b) (Figs. 1a-b, S1b-j, and Table S2). After CODEX imaging we performed hematoxylin and eosin (H\&E) staining on the same tissue sections. We observed excellent morphological correlation between fluorescent markers and H\&E images, exemplified by cytokeratin positivity in the epithelium, CD19 staining of the B cell follicles and CD1c expression in the associated antigen-presenting cells, CD3 staining in the surrounding T cell zone, CD15-positive clusters of granulocytes, and Ki-67 staining of the proliferating cells in germinal centers and basal epithelia (Fig. 1b). Cellular segmentation and fluorescent marker quantification resulted in spatially resolved single-cell data that was used for downstream analysis (Fig. 1c). Using unsupervised X-shift clustering ${ }^{20}$ and manual cluster curation based on marker expression profiles, tissue localization, and tissue morphology, we identified 13 distinct cell-type clusters in our samples (Figs. 1d-e, S2 and S3, and Methods). These included eight distinct immune cell clusters and two epithelial clusters, as well as smooth muscle, mixed stroma, and vasculature clusters. The immune cell clusters consisted of adaptive and innate immune cell types, including three types of T cells, B cells, plasma cells and dendritic cells (DCs), neutrophils, and granulocytes (Figs. 1d-e, S2 and S3). Unsupervised clustering results were compared to manual gating of similar cell populations based on the marker expression profiles, and the frequencies of cell types identified by both methods showed a strong correlation (Figure S4a-c). Of these cell types, we observed Mayo Disease Severity Score-dependent statistically significant changes in the frequencies of T cells, epithelium, plasma cells, granulocytes, DCs, and intraepithelial T cells (Fig. 1f, S4c). For downstream analyses, we chose cell types identified by unsupervised clustering. To verify the clusters and assess their 
spatial distribution in the tissue, we graphically represented the clustering results as Voronoi diagrams colored by cell type and overlaid selected clusters on the H\&E-stained images (Fig. 1g-i). In sum, by CODEX tissue imaging and computational single-cell identification, we created a high-dimensional spatial atlas of the inflammatory microenvironment in UC patients treated or not with TNFi, which can be used for biomarker discovery and clinical correlation using a cloud-based online tool that we call Explorer (Anonymous username for editor/reviewer: reviewer; password: Ulcerativecolitis1!).

\section{Immune cell contacts reorganize gut tissue architecture and contribute to UC disease heterogeneity}

Both the frequencies of epithelial, stromal, and immune cell types in the colonic microenvironment, as well as their spatial organization, should provide insights into how various cell types and their interactions influence UC development, progression, and therapeutic response. A simple way of analyzing spatial organization of cell types in tissue is to compute their pairwise contacts. Analysis of pairwise contacts in images of samples from healthy controls and 14 patients who had not been treated with a $\mathrm{TNFi}$ at the time of biopsy revealed that a spectrum of colonic and immune cell contacts contribute to tissue organization (Fig. 2a). The healthy controls clustered together and were defined by enriched contacts between epithelial cells and stromal cells (Fig. 2a, 6 bottom rows). Interestingly, cellular contacts in UC patient samples showed significant variability that was not strongly associated with Mayo Score. In most patient samples, we observed relative enrichment for paired cell-cell contacts between innate immune cells (e.g., patient UC8; Fig. 2a, center rows), whereas in a minority of patient samples, we observed enrichment for contacts between adaptive immune cells (e.g., patient UC14a; Fig. 2a, top 3 rows). We visualized the cell-type clusters for each patient using Voronoi diagrams (Fig. 2b) and plotted the frequencies of pairwise cell-cell contacts using circular "connectivity" diagrams (Fig. 2c) ${ }^{23}$. The most frequent cell-type clusters and contacts in UC patients were immune related. For example, in patient UC8, T cells were the most frequent cell type, followed by stroma, plasma cells, granulocytes, neutrophils, and vasculature. Epithelial cells were only the seventh most abundant cell-type cluster, which reflects their destruction and replacement by the immune infiltrate during active UC (Figs. 2b-c, middle panels). The cell-cell connectivity map for patient UC14a revealed T cells predominantly connected to B cells and stroma, whereas cell-cell contacts to and in-between innate immune cell types, especially neutrophils, were less frequent (Figs. 2b-c, top panels). We hypothesized that the observed heterogeneity between cellcell contacts in UC patients contributes to differences in higher-order tissue organization and diverging disease states and therapy responses.

\section{Characteristic cellular neighborhoods of the UC immune microenvironment are conserved across patients and are associated with immune cell functional state}

We previously showed that in addition to pairwise cell-cell contacts, higher-order tissue structures such as $\mathrm{CNs}$ provide important information with regards to disease status and therapy response ${ }^{24}$. CNs can be seen as functional subunits inside intact tissue. We performed $\mathrm{CN}$ analysis on the entire UC patient dataset and identified $10 \mathrm{CNs}$ common to all patients (Fig. 2d). Among these were a CN highly enriched in $B$ cells and follicular T cells, which we termed the B cell follicle (CN-9); a CN moderately enriched in $B$ cells, follicular T cells, and T cells, termed lymphoid aggregate (CN-2); a mixed immune CN (CN-4); a 
basal epithelium $\mathrm{CN}$ enriched in epithelial cells, proliferating $\left(\mathrm{Ki}-67^{+}\right)$epithelial cells, intraepithelial T cells, and other immune cells (CN-8); a luminal epithelium $\mathrm{CN}$ with a composition similar to $\mathrm{CN}-8$ but spatially distinct (CN-1); a lamina propria CN enriched in plasma cells and DCs (CN-5); a granulocyte-enriched CN (CN-3); a CN enriched in vasculature, smooth muscle, and neutrophils, which we termed inflamed vasculature (CN-7); a CN enriched in stroma, neutrophils, and other immune cells, which we termed inflamed stroma (CN-0); and a non-inflamed stroma CN (CN-6) (Fig. 2d-e). Significant increases were seen in granulocyte (CN-3), mixed immune ( $\mathrm{CN}-4)$, and lamina propria ( $\mathrm{CN}-5)$ neighborhoods in association with increasing Mayo Score. Unexpectedly, the lymphoid aggregate (CN-2) neighborhood increased through Mayo Score 2, then decreased from Mayo Score 2 to Mayo Score 3. Significant decreases were observed for luminal epithelium (CN-1) and basal epithelium (CN-8) as Mayo Score increased, consistent with healthy gut tissue destruction and the trends observed at the cell frequency level (Figure S5).

In addition to determining the frequencies of CNs across patient groups, we also analyzed their "functional states" as previously described ${ }^{24}$. CN functional states are determined based on the local enrichment with one or more specific cell types expressing certain functional markers, and their analysis provides a measure of how a given CN's characteristics influence cellular function during disease progression and treatment. Specifically, we were interested in how TNFi treatment influenced the distribution of TNFR2 on various cell types and how this affected functional states. We found that $\mathrm{CN}-7$ (inflamed vasculature) had a significantly higher frequency of $\mathrm{TNFR}^{+}$neutrophils as compared to $\mathrm{CN}-0$ and $\mathrm{CN}-4$, the two other neighborhoods enriched the most for neutrophils (Fig. 2f-g). Similarly, PD1 ${ }^{+} \mathrm{T}_{\text {cell }}$ frequencies varied greatly depending on whether a $T$ cell was found in a B cell follicle neighborhood (CN9), a lymphoid aggregate (CN-2), or a mixed immune neighborhood (CN-4) (Fig. 2h-i). This suggests an important role for $\mathrm{CNs}$ in the functional states of individual cells.

\section{Innate immune cell populations persist during TNFi therapy}

Identification of the biological basis for the resistance to TNFi might allow for a priori identification of UC patients who will be resistant to TNFi treatment and inform selection of an alternative therapy. To understand the mechanisms that underlie resistance to TNFi, we created a framework to assess architectural changes associated with gut inflammation during disease progression and mucosal healing in response to TNFi treatment. These architectural changes were first depicted utilizing minimum spanning trees, where average marker expression is visualized as a function of cell type. We observed that the expression level of the co-stimulatory TNF receptor 4-1BB (also known as TNFRSF9 or CD137), a marker of T cell activation implicated in UC inflammation ${ }^{26}$, increased with disease severity, and levels decreased during treatment except for in neutrophils and proliferative epithelium (Fig. 3a). Architectural changes were further quantified using cell-type frequency. TNFi responsiveness was primarily defined by mucosal healing (e.g., epithelium recovery) (Fig. 3a, S6). Indeed, TNFi therapy had substantial effects on the frequencies of adaptive immune cell types (Fig. 3b-c). Specifically, we observed statistically significant reductions in T cell frequency and increases in epithelium in treated vs. untreated Mayo 2 patients. Although B cell levels appeared to decrease upon treatment, these differences did not reach statistical significance due to the high variability of B cell frequencies in UC patients. Compared to their 
adaptive counterparts, there were no strong trends or significant changes in frequencies of innate immune cells in the face of TNF blockade.

\section{TNFi therapy rewires adaptive immune cell contacts}

Given the observation that TNFi therapy decreased adaptive immune cell frequencies such as T cells and $B$ cells, we sought to determine whether the treatment rewired immune cell contacts. We observed that treatment altered contacts between $T$ cells and other adaptive immune cells (e.g., $T$ cell - T cell, $T$ cell - B cell). This was similarly true for contacts between T cells with gut stroma and epithelium. (Fig. 3d-e). In contrast, $T$ cell contacts with innate immune cells such as granulocytes were completely unchanged by treatment (Fig. 3d-e). Homotypic innate immune cell contacts such as granulocyte granulocyte contacts did not differ with or without TNFi therapy either. This rewiring of only certain immune cell contacts suggested that TNFi treatment has spatially dependent effects on inflammatory cellular neighborhoods in the gut.

\section{Innate immune neighborhoods are resistant to TNFi therapy}

We next examined changes to $\mathrm{CNs}$ associated with disease severity, progression, and recovery to determine whether observed changes in cell frequencies and cell-cell contacts are correlated with higherorder architectural changes. To visualize reorganization of $\mathrm{CNs}$ during disease progression and therapy, we performed principal component analysis (PCA) on the $\mathrm{CN}$ frequencies for healthy controls and untreated patients to map the trajectories associated with disease progression (Fig. 3f). When CNs weights were plotted onto principal components 1 and 2, cluster centroids separated into roughly 4 quadrants, with disease severity increasing on average along a clockwise trajectory. Healthy controls clustered in the upper right quadrant and were associated with basal and luminal epithelium (CN-8 and $\mathrm{CN}-1$, respectively). Mayo Score 1 patients, although scarce in our data set, were similar to healthy controls but the population centroid was pulled into quadrant 2 due to increased contributions from stroma (CN-6) and inflamed stroma (CN-0) neighborhoods (Fig. 3f, Q2). Patients with Mayo Scores 2 and 3 showed considerable overlap with one another, although patients within these groups separated into quadrants 3 or 4 driven by differences in their underlying $\mathrm{CN}$ organization. Patients in quadrant 3 were characterized by increased contributions from adaptive immune $\mathrm{CNs}$ such as lymphoid aggregates (CN2) and $B$ cell follicles (CN-9), whereas patients in quadrant 4 were characterized by innate immune $\mathrm{CNs}$ such as the granulocyte CN (CN-3) (Fig. 3f, Q3 and Q4).

Comparing currently TNFi-treated with untreated patients of the same endoscopic score (Mayo 2) highlighted changes in tissue architecture associated with TNFi treatment, even when the clinical disease severity was similar (Fig. 3g). The cluster centroid for Mayo 2 TNFi treated patients localized between quadrant 4 and quadrant 1 when projected onto the same principal component axes as before. This suggested that changes with TNFi treatment were predominantly associated with increased epithelial neighborhoods $\mathrm{CN}-1$ and $\mathrm{CN}-8$ (Q1) and decreased contributions from lymphoid aggregate (CN-2) and $\mathrm{B}$ cell follicle (CN-9) neighborhoods (Q3), leading to a shift toward normal-like tissue architectures upon treatment. Notably, lack of change in the granulocyte $\mathrm{CN}(\mathrm{CN}-3, \mathrm{Q} 4)$ prevented Mayo 2 TNFi treated patients from fully returning to the healthy state space (Q1). This suggested that these innate niches may 
be resistant to therapy, whereas adaptive immune neighborhoods normalize during TNFi treatment. Univariate analysis of TNFi-treated patients controlled for Mayo Score 2 demonstrated that adaptive immune CNs lymphoid aggregate and B cell follicle (CN-2 and CN-9, respectively) showed the greatest decrease upon treatment, whereas epithelial CNs like basal epithelium (CN-8) increased (Fig. 3h). In contrast, frequencies of innate immune CNs did not change significantly upon treatment, suggesting potential treatment resistance. Interestingly, we observed that adaptive immune cells, contacts, and CNs were enriched in female patients compared to males (Figure S7), which may underlie the reported higher TNFi treatment response rates in females ${ }^{27}$.

\section{Cellular Voronoi maps predict resistance to TNFi therapy}

Using our spatial tissue atlas, we built a neural network model to predict patient TNFi resistance (Fig. 4a). Unlike cell-type, pairwise cell-cell contact, and $\mathrm{CN}$ analyses, our neural network-based classification system performed an unbiased examination of spatial features across multiple scales. In this approach, we utilized Voronoi representations of our patient samples, which has two advantages: First: using Voronoi diagrams instead of raw images reduces the effects of image variance and therefore minimizes the impact of intra-facility differences in sample preparation and imaging. Second, using cell-type Voronoi maps reduced the dimensionality of our dataset.

Our neural network predicted TNFi resistance with an AUROC of 0.947 (Fig. 4b). It substantially outperformed L1-regularized logistic regression classifiers based on cell types (AUROC $=0.735$ ), cell-cell contacts (AUROC $=0.823)$, and CNs $(A \cup R O C=0.654)$, suggesting that higher-order spatial features or feature interactions underlie resistance (Figure S8). Using our established cell types, cell-cell contacts, and $\mathrm{CNs}$, we identified neutrophils as associated with resistance to TNFi. Confirming our earlier findings regarding innate immune cell (e.g. Granulocytes) persistence in patients treated with TNFi, these differences between subsequent TNFi responders and non-responders were most notably associated with cell-cell contacts involving neutrophils and T cells and between neutrophils and vasculature (Fig. 4c). While neutrophil frequency alone was associated with response, the location of neutrophils within the tissue (e.g. neutrophils-stroma vs neutrophils-vessels) had a significant effect on subsequent TNFi response (Fig. 4d-f).

\section{Discussion}

Ulcerative colitis and other autoimmune diseases are among the most common inflammatory disorders worldwide ${ }^{1,28,29}$. New biological therapies such as TNFi have improved patient quality of life and reduced long-term complications of these chronic diseases. However, due to the toxicities, adverse effects, substantial failure rates, and high treatment costs associated with biologics, clinicians are in need of predictive biomarkers to guide the choice of therapy $y^{9,11,27}$. Recent studies using single-cell technologies such as scRNA-seq and mass cytometry have attempted to identify such biomarkers and differences in the intramucosal immune systems of patients compared to healthy controls; however, these technologies cannot measure tissue architecture and the spatial interactions of cells ${ }^{17,18,30}$. Cellular organization is 
known to provide critical insights into disease progression and treatment response ${ }^{24}$. We therefore reasoned that analyzing the gut architecture in UC patients treated or not with TNFi using highly multiplexed microscopy to interrogate the tissue at multiple levels simultaneously (i.e. at the level of cell type frequencies, cell-cell contacts, and higher-order architectural features such as CNs) should enable new biologic insights.

By taking advantage of CODEX's unique highly multiplexed tissue visualization with single-cell spatial resolution, we were able to analyze the spatial relationships between different cells to achieve important new insights into the underlying architectural pathophysiology of UC. These spatial insights would not have been possible with single cell technologies alone. We used semi-autonomous algorithms to define and map cell types, cell-cell contacts, and neighborhoods in the gut across the UC inflammatory spectrum and created a comprehensive tissue atlas of the UC gut. We created a cloud-based software platform to make this tissue atlas publicly available and queryable. This interface allows the scientific community to explore our dataset, generate, and test additional hypotheses. This atlas enabled the modeling of disease severity and the identification of cellular niches that are TNFi treatment resistant and sex specific.

We also trained a neural network to classify TNFi responders versus non-responders based on Voronoi representations of the cell types in our UC atlas. This novel approach significantly outperformed L1penalized logistic regression models based on cell types, cell-cell contacts, or CNs. L1-penalized logistic regression was selected for comparison as the output (TNFi prediction) is categorical rather than continuous and due to increased interpretability from shrinking many correlation coefficients to zero ${ }^{31}$. Our results recapitulated the known pathogenesis of ulcerative colitis at the single-cell level including observations of increased inflammatory immune cell populations, including plasma cells, granulocytes, and T cells, and provided novel insights into changes in cell-cell interactions and CNs during different stages of disease activity ${ }^{1,3,32}$. Our observations of increased plasma cell and follicular and non-follicular T cell frequencies in UC are consistent with single-cell literature identifying expansions in subsets of plasma cells and tissue-resident memory $T$ cells $^{30}$. Our predictive logistic regression classifiers indicate that proliferative epithelium is critical for recovery, consistent with existing literature ${ }^{33,34}$. Similarly, in line with previous findings, we identify granulocytes and neutrophils as negative predictors of recovery 35 . Whether increased neutrophils are directly pathological or are a surrogate of other pathological factors such as microbial infiltration is unclear ${ }^{36}$. Importantly, our data illuminates the spatial organization of these cell populations, revealing the rewiring of the gut architecture during UC. Significant heterogeneity in cell frequencies and cell-cell contacts was observed across our cohort, yet conserved CNs were identified. These CNs impacted the functional states of cells that resided within them. For example, neutrophils in inflamed vessel CNs were more likely to express the TNFi drug target TNFR2 than neutrophils found elsewhere in the tissue. Furthermore, we found that innate cellular niches were unchanged during therapy, whereas adaptive immune cell niches were significantly rewired. Females in our study were enriched in these responsive adaptive immune CNs compared to males, providing a potential biological underpinning to previously observed and unaccounted for sex differences in response to TNFi inhibitors 37,38 . 
The advances presented here have substantial potential, pending validation with larger patient cohorts, to guide selection of optimal therapeutic strategies for individual UC patients. Furthermore, more generally, this study offers insights into the central biological question of how higher-order tissue properties emerge from cellular organization. Although we have identified architectural motifs associated with TNFi resistance in UC in this patient cohort, it is still unclear how these motifs mechanistically lead to TNFi resistance and how these might be therapeutically altered in order to regain TNFi responsiveness. Our data suggests that combination therapies targeting innate immune cells such as neutrophils merit further investigation. The presence of these motifs provide insights into the cellular basis of inflammation, though it is still uncertain as to whether these motifs are inherently pathological, or if they are required components of a normal inflammatory response and it is only their aberrant persistence that is pathological. The tools we have developed are broadly applicable for study of other immune-driven diseases, and the potential roles of these motifs in other autoinflammatory disorders should be further studied.

This was a retrospective translational study designed primarily for technical feasibility and hypothesis generation. The biological insights described in this work and their correspondence with clinical phenotypes must be tested in properly powered and controlled prospective studies. Our panel was used to identify 13 cell types in the gut, but, notably, several important classes of cells to gut mucosal immunology were not identified including regulatory $T$ cells, mast cells, natural killer cells, and macrophages. This will be addressed in further studies by refinement of the biomarker panel used for celltype enumeration, with a focus on resolving $T$ cell subsets, which have recently been implicated in $\mathrm{UC}^{30,39}$. Further classification of cell type based on the surrounding neighborhood may also refine analysis. Furthermore, there were a number of functional markers included in this study that were not fully analyzed within the scope of this work, despite their known roles and importance in IBD such as HIF1a. Further studies will aim at functional analysis of CNs. The novel analysis frameworks introduced here, such as the utilization of neural networks adapted from the computer vision field to make clinical response predictions based on cell type Voronoi diagrams, will need to be rigorously tested. We believe this methodology represents a powerful new approach, but questions remain, such as how the model performs when investigating larger or smaller tissue patch sizes, how many cell types are necessary to maintain classification accuracy, and the number of biomarkers needed for a particular clinical classification task. The UC spatial tissue atlas and analysis tools described herein provide a framework that future studies will build upon to realize precision medicine for patients with UC.

\section{Declarations}

\section{CONFLICTS OF INTEREST}

S.R. has received research support from Genentech. A.T.M. and S. Bodapati are cofounders of, U.T. is an employee of, and C.M.S. is a scientific advisor to Enable Medicine, LLC. G.P.N. has equity in and is a scientific board member of Akoya Biosciences, Inc. B.S.B has received research grants from Prometheus Biosciences and consulting fees from Pfizer, Bristol Myers Squibb, and Takeda unrelated to this topic. 
S.E.S. has received consulting fees from Prometheus Biosciences, Pfizer, Takeda, Gilead, Janssen, Celgene, and Surrozen unrelated to this topic. W.J.S. has received research grants from Abbvie, Abivax, Arena Pharmaceuticals, Boehringer Ingelheim, Celgene, Genentech, Gilead Sciences, GlaxoSmithKline, Janssen, Lilly, Pfizer, Prometheus Biosciences, Seres Therapeutics, Shire, Takeda, Theravance Biopharma; consulting fees from Abbvie, Abivax, Admirx, Alfasigma, Alimentiv (Robarts Clinical Trials, owned by Health Academic Research Trust [HART]), Alivio Therapeutics, Allakos, Amgen, Applied Molecular Transport, Arena Pharmaceuticals, Bausch Health (Salix), Beigene, Bellatrix Pharmaceuticals, Boehringer Ingelheim, Boston Pharmaceuticals, Bristol Myers Squibb, Celgene, Celltrion, Cellularity, Cosmo Pharmaceuticals, Escalier Biosciences, Equillium, Forbion, Genentech/Roche, Gilead Sciences, Glenmark Pharmaceuticals, Gossamer Bio, Immunic (Vital Therapies), Index Pharmaceuticals, Intact Therapeutics, Janssen, Kyverna Therapeutics, Landos Biopharma, Lilly, Oppilan Pharma, Otsuka, Pandion Therapeutics, Pfizer, Progenity, Prometheus Biosciences, Protagonists Therapeutics, Provention Bio, Reistone Biopharma, Seres Therapeutics, Shanghai Pharma Biotherapeutics, Shire, Shoreline Biosciences, Sublimity Therapeutics, Surrozen, Takeda, Theravance Biopharma, Thetis Pharmaceuticals, Tillotts Pharma, UCB, Vedanta Biosciences, Ventyx Biosciences, Vimalan Biosciences, Vivelix Pharmaceuticals, Vivreon Biosciences, Zealand Pharma; and stock or stock options from Allakos, BeiGene, Gossamer Bio, Oppilan Pharma, Prometheus Biosciences, Progenity, Shoreline Biosciences, Ventyx Biosciences, Vimalan Biosciences. Spouse: Iveric Bio - consultant, stock options; Progenity - stock; Oppilan Pharma - consultant, stock options; Prometheus Biosciences - employee, stock options; Ventyx Biosciences - stock options; Vimalan Biosciences - stock options. The other authors declare no competing interests.

\section{AUTHOR CONTRIBUTIONS}

Conceptualization: A.T.M., C.M.S., and S.R.; Methodology: A.T.M., S. Black, C.M.S., and S.R.; Software: A.T.M., U.T., J.C., G.L.B., and S.S.B.; Validation: S. Black and C.M.S.; Formal Analysis: A.T.M., D.R.H., and C.M.S.; Investigation: A.T.M., D.R.H., C.M.S., and S.R.; Resources: E.C.C., S. Bodapati, J.C., S.E.S., B.S.B., W.S.J., J.T.C., and S.R.; Writing - Original Draft: A.T.M., D.R.H., C.M.S., and S.R.; Writing - Review and Editing: A.T.M., D.R.H., S.S.B., B.S.B., J.T.C., G.P.N., C.M.S., and S.R.; Supervision: C.M.S. and S.R.; Project Administration: E.C.C.; Funding acquisition: S.S.G., G.P.N., C.M.S., and S.R.

\section{ACKNOWLEDGMENTS}

We thank the patients for their consent to use their tissues for research. This work was primarily supported by the Kenneth Rainin Foundation (2018 - 575 to S.R.). Further support was provided by the US National Institutes of Health (2U19AI057229-16, 5P01HL10879707, 5R01GM10983604, 5R33CA18365403, 5U01Al101984-07, 5UH2AR06767604, 5R01CA19665703, 5U54CA20997103, 5F99CA212231-02, 1F32CA233203-01, 5U01Al140498-02, 1U54HG010426-01, 5U19Al100627-07, 1R01HL120724-01A1, R33CA183692, R01HL128173-04, 5P01Al131374-02, 5UG3DK114937-02, 1U19AI135976-01, IDIQ17X149, 1U2CCA233238-01, 1U2CCA233195-01 to G.P.N.) and by the NIDDKfunded San Diego Digestive Diseases Research Center (P30DK120515). C.M.S. was supported by the Swiss National Science Foundation (P300PB_171189, P400PM_183915). S.S.B. was supported by a 
Stanford Bio-X Interdisciplinary Graduate Fellowship and Stanford's Bioengineering Department. G.L.B was supported by an NIH T32 Fellowship (5T32AI007290-34).

\section{References}

1. Ungaro, R., Mehandru, S., Allen, P. B., Peyrin-Biroulet, L. \& Colombel, J.-F. Ulcerative colitis. The Lancet 389, 1756-1770 (2017).

2. Cooper, G. S., Bynum, M. L. K. \& Somers, E. C. Recent Insights in the Epidemiology of Autoimmune Diseases: Improved Prevalence Estimates and Understanding of Clustering of Diseases. $J$ Autoimmun 33, 197-207 (2009).

3. Neurath, M. F. Targeting immune cell circuits and trafficking in inflammatory bowel disease. Nat Immunol 20, 970-979 (2019).

4. Dekker, E., Tanis, P. J., Vleugels, J. L. A., Kasi, P. M. \& Wallace, M. B. Colorectal cancer. The Lancet 394, 1467-1480 (2019).

5. Olén, O. et al. Colorectal cancer in Crohn's disease: a Scandinavian population-based cohort study. The Lancet Gastroenterology \& Hepatology S2468125320300054 (2020) doi:10.1016/S24681253(20)30005-4.

6. Neurath, M. F. Current and emerging therapeutic targets for IBD. Nat Rev Gastroenterol Hepato/ 14, 269-278 (2017).

7. Feuerstein, J. D. et al. AGA Clinical Practice Guidelines on the Management of Moderate to Severe Ulcerative Colitis. Gastroenterology 158, 1450-1461 (2020).

8. Axelrad, J. E., Lichtiger, S. \& Yajnik, V. Inflammatory bowel disease and cancer: The role of inflammation, immunosuppression, and cancer treatment. World J Gastroentero/ 22, 4794-4801 (2016).

9. Kopylov, U. \& Seidman, E. Predicting durable response or resistance to antitumor necrosis factor therapy in inflammatory bowel disease. Therap Adv Gastroenterol 9, 513-526 (2016).

10. Ben-Horin, S. \& Chowers, Y. Tailoring anti-TNF therapy in IBD: drug levels and disease activity. Nat Rev Gastroenterol Hepatol 11, 243-255 (2014).

11. Abraham, C., Dulai, P. S., Vermeire, S. \& Sandborn, W. J. Lessons Learned From Trials Targeting Cytokine Pathways in Patients With Inflammatory Bowel Diseases. Gastroenterology 152, 374-388.e4 (2017).

12. Gaujoux, R. et al. Cell-centred meta-analysis reveals baseline predictors of anti-TNFa non-response in biopsy and blood of patients with IBD. Gut 68, 604-614 (2019).

13. Colombel, J.-F. et al. The safety of vedolizumab for ulcerative colitis and Crohn's disease. Gut 66, 839-851 (2017).

14. Borg-Bartolo, S. P., Boyapati, R. K., Satsangi, J. \& Kalla, R. Precision medicine in inflammatory bowel disease: concept, progress and challenges. F1000Res 9, (2020). 
15. Lehmann, F. S., Burri, E. \& Beglinger, C. The role and utility of faecal markers in inflammatory bowel disease. Therap Adv Gastroentero/ 8, 23-36 (2015).

16. Ding, N. S., Hart, A. \& De Cruz, P. Systematic review: predicting and optimising response to anti-TNF therapy in Crohn's disease - algorithm for practical management. Aliment Pharmacol Ther 43, 30-51 (2016).

17. Rubin, S. J. S. et al. Mass cytometry reveals systemic and local immune signatures that distinguish inflammatory bowel diseases. Nat Commun 10, 2686 (2019).

18. Martin, J. C. et al. Single-Cell Analysis of Crohn's Disease Lesions Identifies a Pathogenic Cellular Module Associated with Resistance to Anti-TNF Therapy. Cell 178, 1493-1508.e20 (2019).

19. Haberman, Y. et al. Ulcerative colitis mucosal transcriptomes reveal mitochondriopathy and personalized mechanisms underlying disease severity and treatment response. Nat Commun 10, (2019).

20. Jackson, H. W. et al. The single-cell pathology landscape of breast cancer. Nature $578,615-620$ (2020).

21. Damond, N. et al. A Map of Human Type 1 Diabetes Progression by Imaging Mass Cytometry. Cell Metab 29, 755-768.e5 (2019).

22. Bodenmiller, B. Multiplexed Epitope-Based Tissue Imaging for Discovery and Healthcare Applications. Cell Systems 2, 225-238 (2016).

23. Goltsev, Y. et al. Deep Profiling of Mouse Splenic Architecture with CODEX Multiplexed Imaging. Cell 174, 968-981.e15 (2018).

24. Schürch, C. M. et al. Coordinated Cellular Neighborhoods Orchestrate Antitumoral Immunity at the Colorectal Cancer Invasive Front. Cell S0092867420308709 (2020) doi:10.1016/j.cell.2020.07.005.

25. Fine, S., Papamichael, K. \& Cheifetz, A. S. Etiology and Management of Lack or Loss of Response to Anti-Tumor Necrosis Factor Therapy in Patients With Inflammatory Bowel Disease. 10.

26. Rabe, H. et al. Distinct patterns of naive, activated and memory $T$ and $B$ cells in blood of patients with ulcerative colitis or Crohn's disease. Clinical \& Experimental Immunology 197, 111-129 (2019).

27. Hyrich, K. L., Watson, K. D., Silman, A. J., Symmons, D. P. M. \& The BSR Biologics Register. Predictors of response to anti-TNF- therapy among patients with rheumatoid arthritis: results from the British Society for Rheumatology Biologics Register. Rheumatology 45, 1558-1565 (2006).

28. Davidson, A. 080201 Autoimmune Diseases. The New England Journal of Medicine 12.

29. Hunter, T. M. et al. Prevalence of rheumatoid arthritis in the United States adult population in healthcare claims databases, 2004-2014. Rheumatol Int 37, 1551-1557 (2017).

30. Boland, B. S. et al. Heterogeneity and clonal relationships of adaptive immune cells in ulcerative colitis revealed by single-cell analyses. Science Immunology 5, (2020).

31. Tibshirani, R. Regression Shrinkage and Selection Via the Lasso. Journal of the Royal Statistical Society: Series B (Methodological) 58, 267-288 (1996). 
32. Smillie, C. S. et al. Intra- and Inter-cellular Rewiring of the Human Colon during Ulcerative Colitis. Cell 178, 714-730.e22 (2019).

33. Farr, L. et al. CD74 Signaling Links Inflammation to Intestinal Epithelial Cell Regeneration and Promotes Mucosal Healing. Cellular and Molecular Gastroenterology and Hepatology 10, 101-112 (2020).

34. Kattah, M. et al. P-285 A20 and ABIN-1 Cooperate to Preserve Intestinal Epithelial Homeostasis. Inflamm Bowel Dis 23, S92-S92 (2017).

35. Zezos, P. et al. Severe eosinophilic infiltration in colonic biopsies predicts patients with ulcerative colitis not responding to medical therapy. Colorectal Disease 16, 0420-0430 (2014).

36. Wéra, O., Lancellotti, P. \& Oury, C. The Dual Role of Neutrophils in Inflammatory Bowel Diseases. Journal of Clinical Medicine 5, 118 (2016).

37. Laganà, B. et al. Sex Differences in Response to TNF-Inhibiting Drugs in Patients With Spondyloarthropathies or Inflammatory Bowel Diseases. Front. Pharmacol. 10, (2019).

38. Rusman, T. et al. Gender differences in retention rate of tumor necrosis factor alpha inhibitor treatment in ankylosing spondylitis: a retrospective cohort study in daily practice. Int J Rheum Dis 21, 836-842 (2018).

39. Imam, T., Park, S., Kaplan, M. H. \& Olson, M. R. Effector T Helper Cell Subsets in Inflammatory Bowel Diseases. Front. Immunol. 9, (2018).

40. Satsangi, J., Silverberg, M. S., Vermeire, S. \& Colombel, J.-F. The Montreal classification of inflammatory bowel disease: controversies, consensus, and implications. Gut 55, 749-753 (2006).

41. Samusik, N., Good, Z., Spitzer, M. H., Davis, K. L. \& Nolan, G. P. Automated mapping of phenotype space with single-cell data. Nature methods 13, 493 (2016).

\section{Figures}



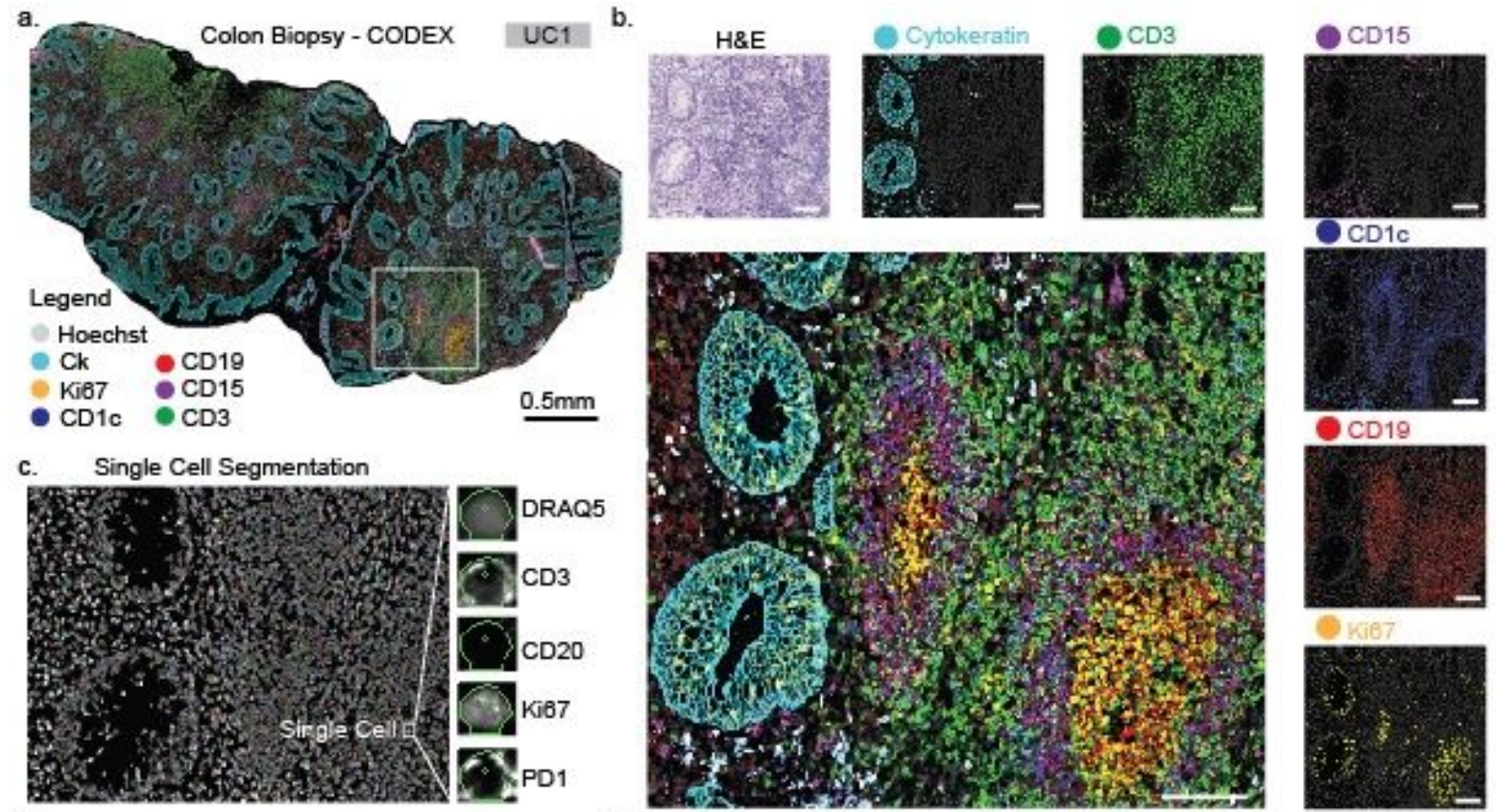

CD19

d.
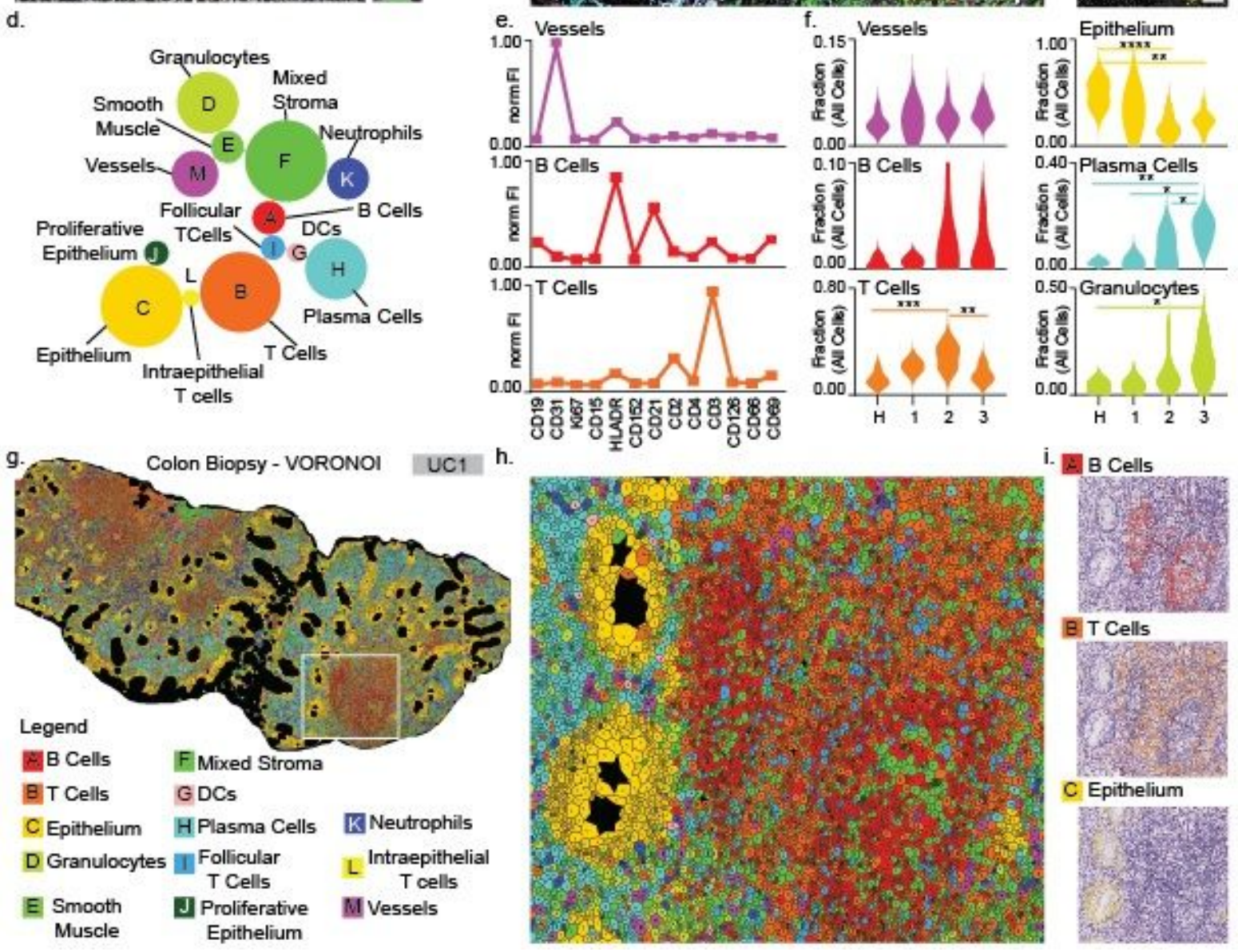

\section{Figure 1}

A spatial atlas of the UC inflammatory microenvironment. 
a.

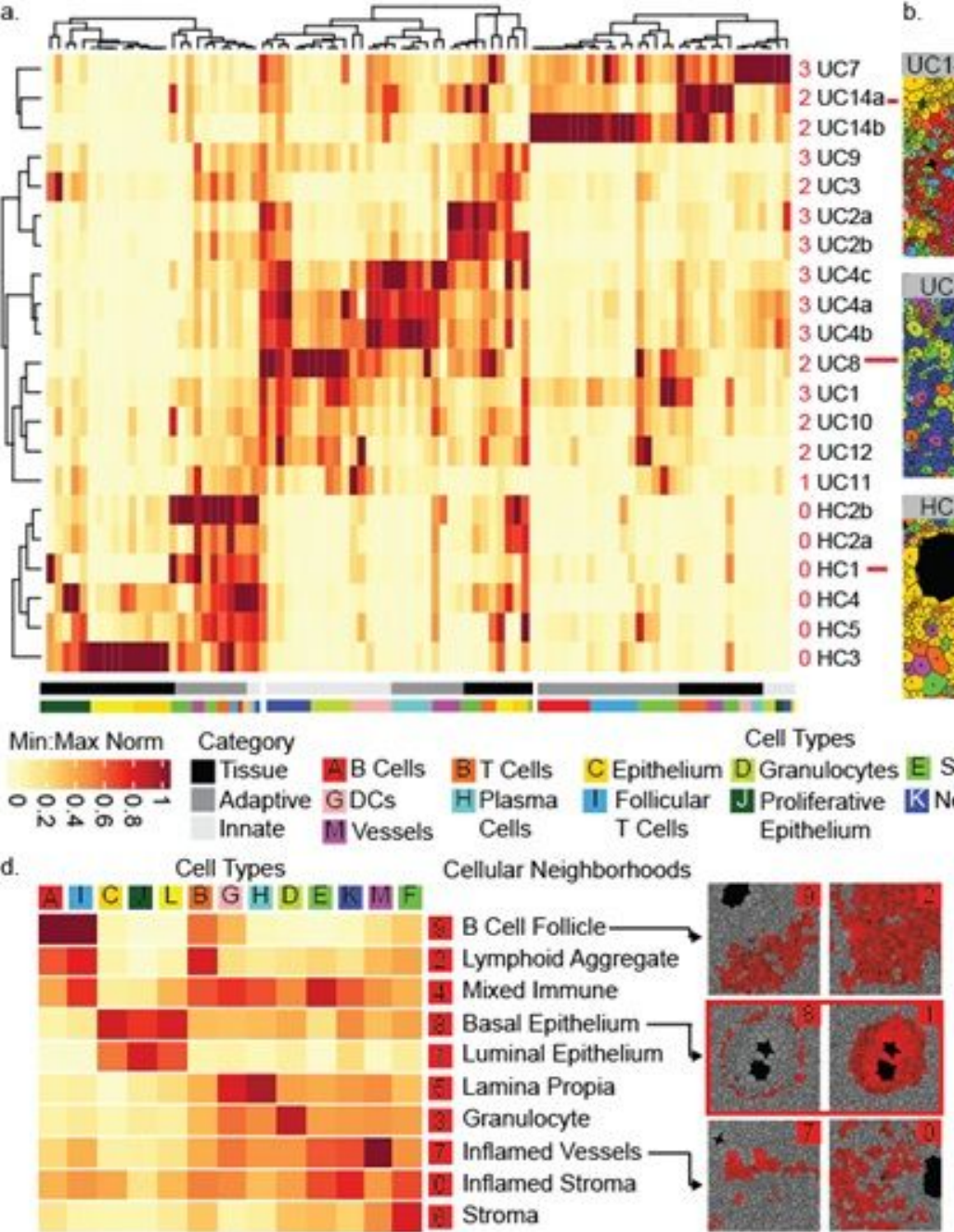

Cell Map

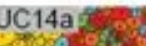

(c)

ins
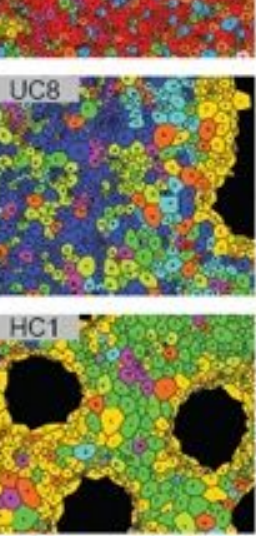

c. Cell Contacts $\mathrm{UC14}_{K}$. ${ }^{B} \mathrm{P}$ of
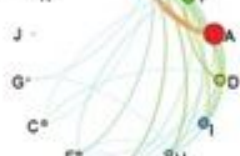

UC8, $L{ }^{\circ} \bigcirc$

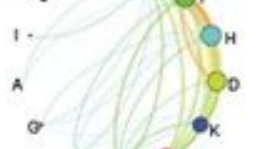

e $\bullet_{M}$

$\mathrm{HCl}_{G}$ a. ${ }^{\circ} \mathrm{O}_{\mathrm{F}}$

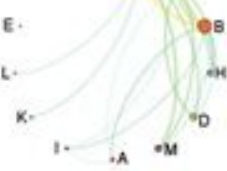

Mixed

Counts e. Cellular Neighborhood Inflamed Vessels UC1

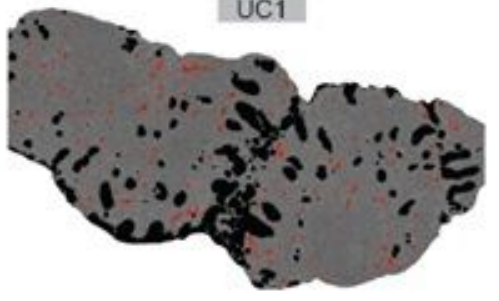

f
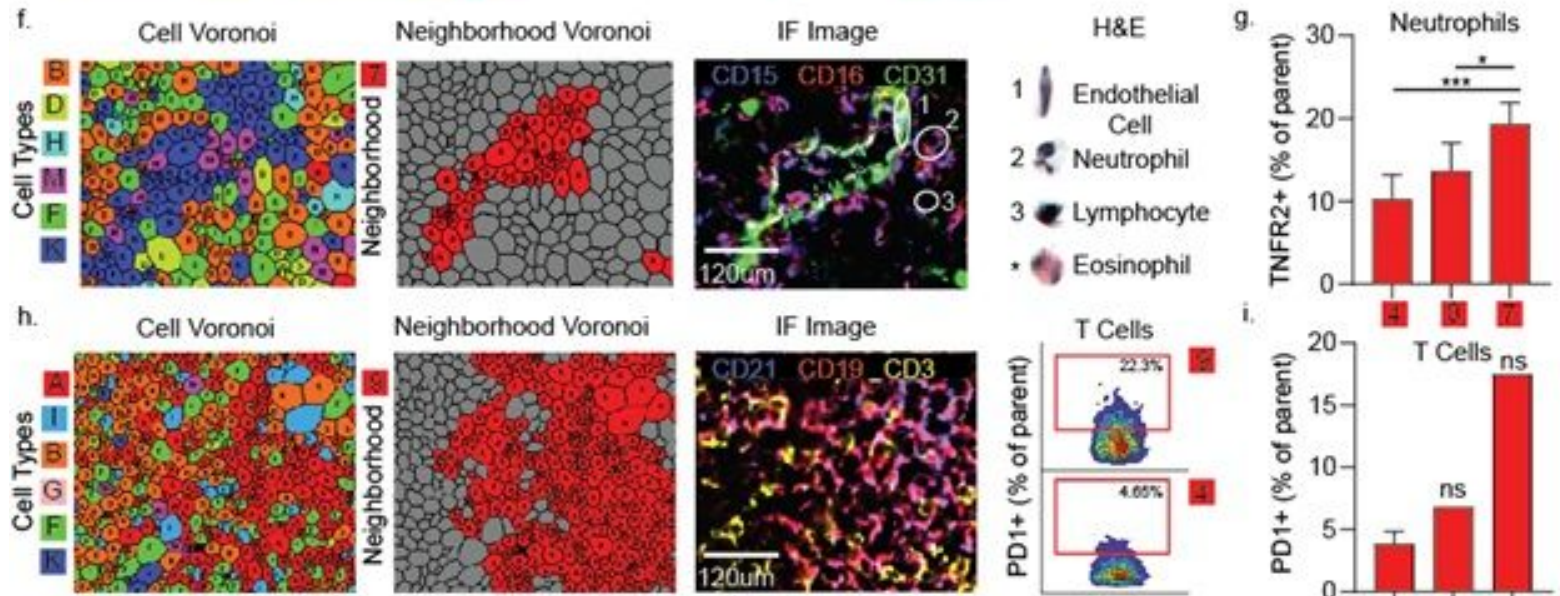

Neighborhcod Voronoi
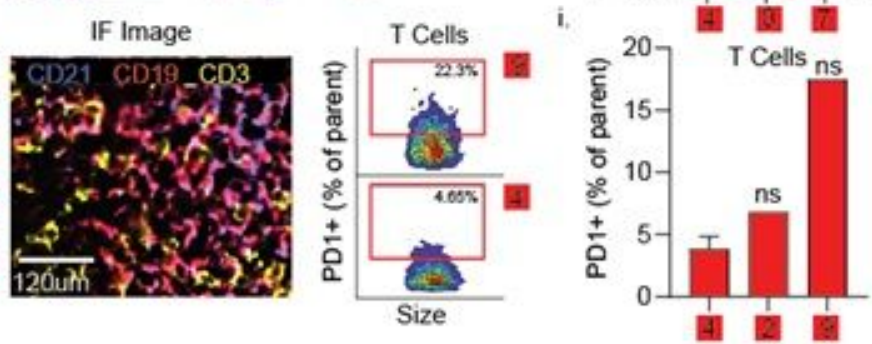

\section{Figure 2}

Immune cell contacts disrupt gut architecture and generate inflammatory niches during UC. 
a.

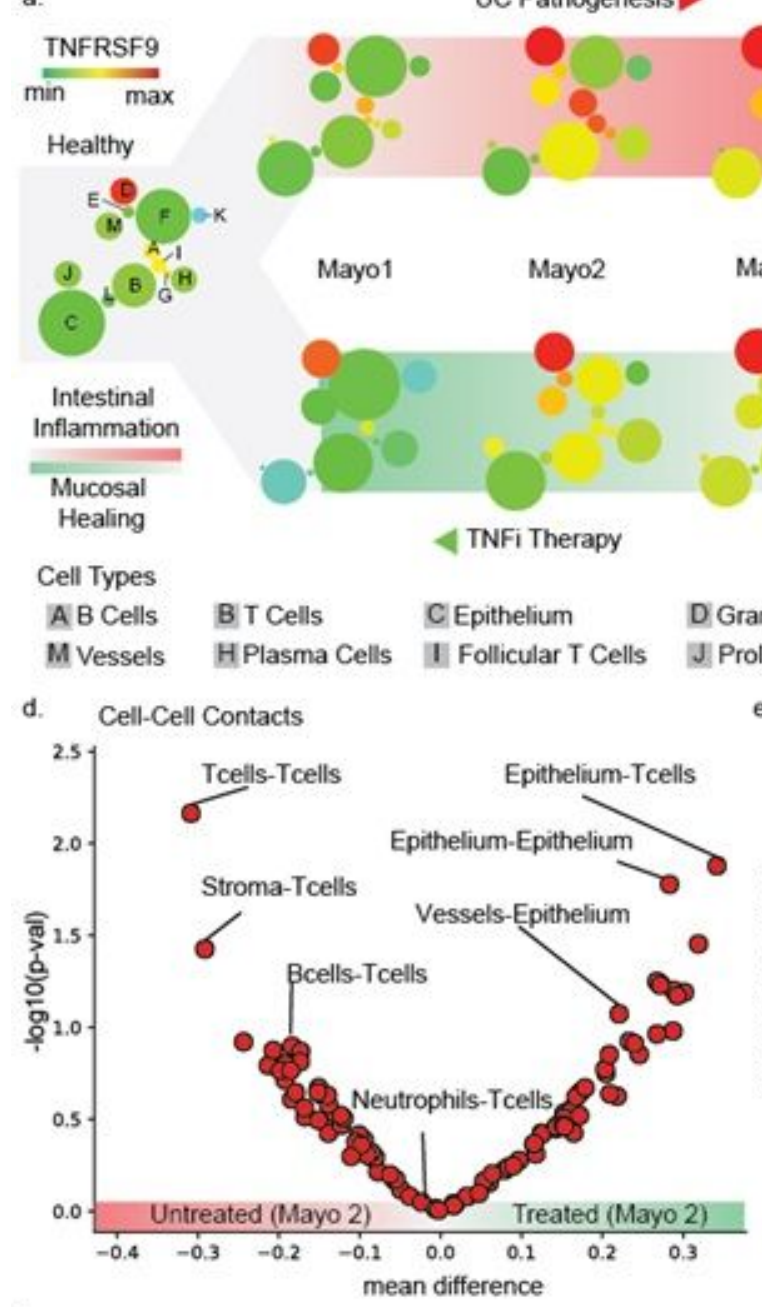

f.

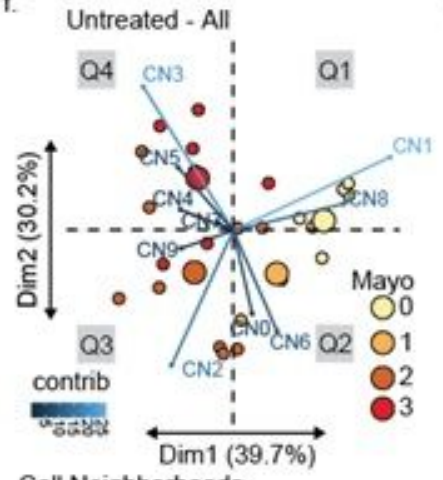

Cell Neighborhoods

g. Treated vs Untreated - Mayo 2

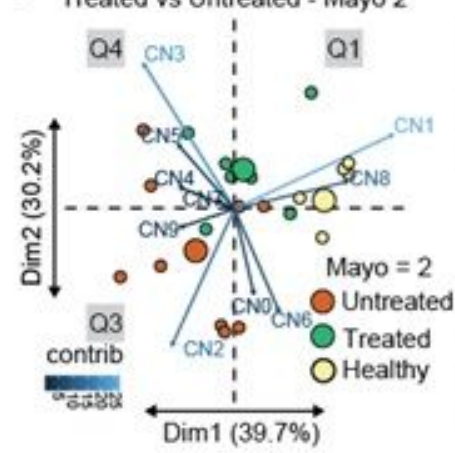

h.
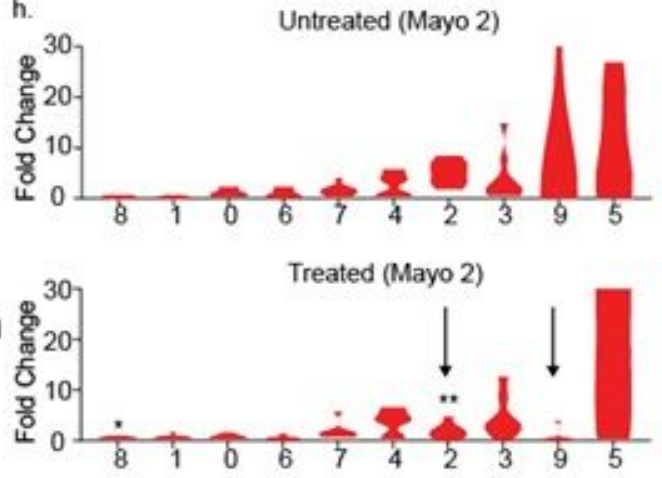

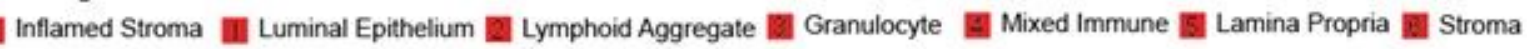
Inflamed Vessels 1 Basal Epithelium B B Cell Follicle

\section{Figure 3}

TNFi therapy shifts adaptive immune cells, contacts, and neighborhoods toward homeostasis. 


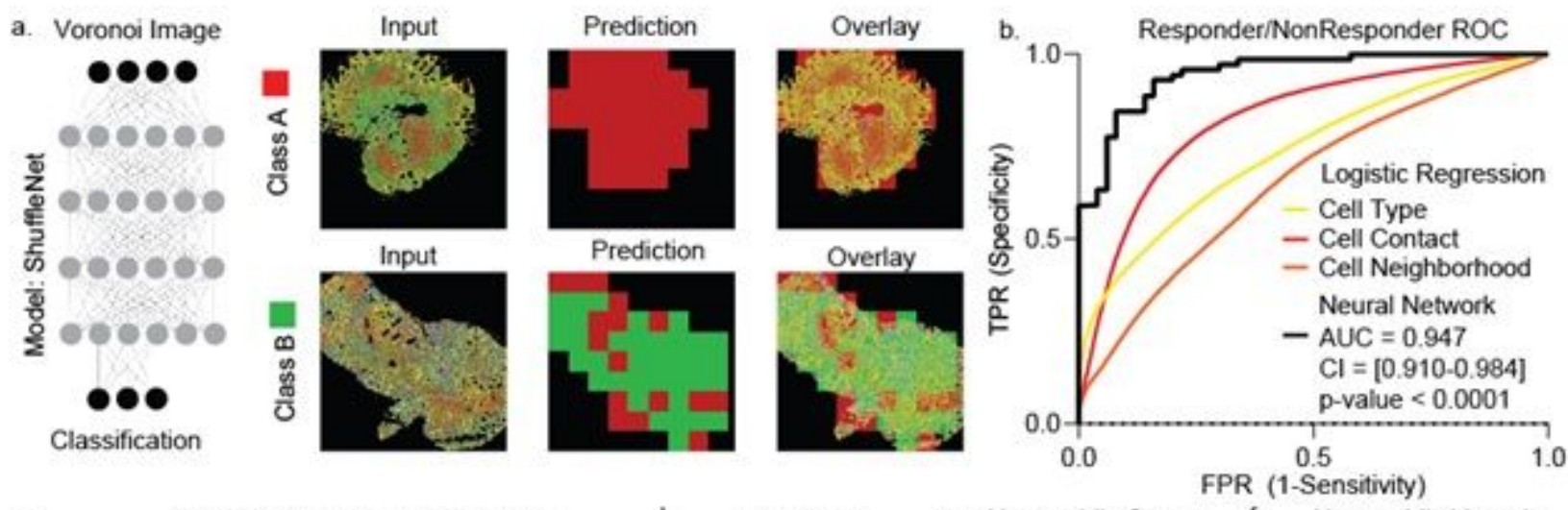

c.

d. Neutrophils

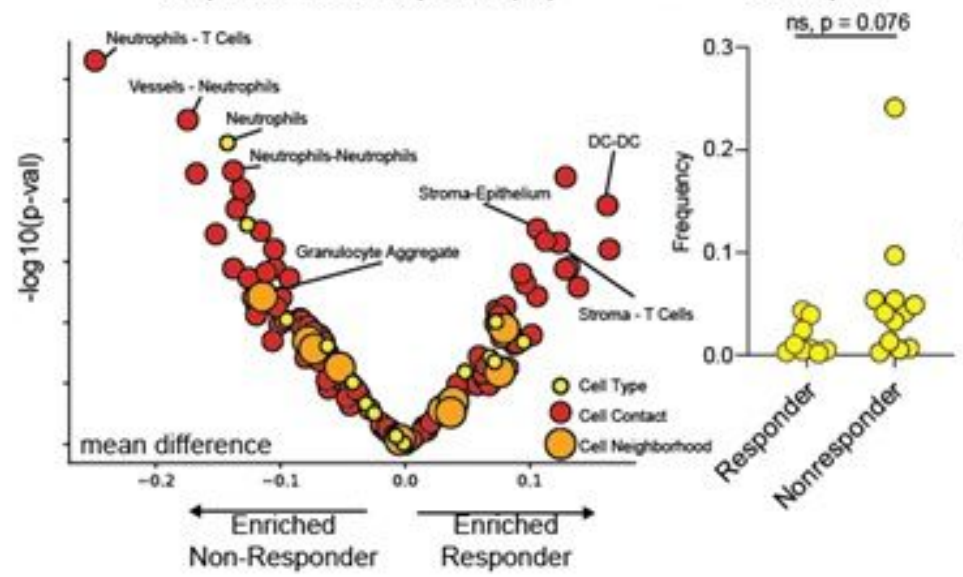

e. Neutrophils-Stroma f. Neutrophils-Vessels
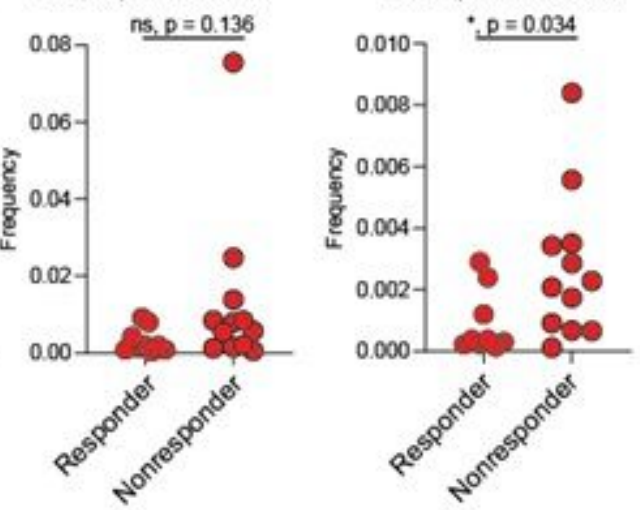

Figure 4

Computer vision and cellular Voronoi maps enable prediction of response to TNFi therapy.

\section{Supplementary Files}

This is a list of supplementary files associated with this preprint. Click to download.

- IBDPrecisionMedicineCODEXSupplement1.docx 